Western University

Scholarship@Western

Medical Biophysics Publications

Medical Biophysics Department

$2-12-2015$

\title{
Right ventricular segmentation in cardiac MRI with moving mesh correspondences.
}

Kumaradevan Punithakumar

Michelle Noga

Ismail Ben Ayed

Pierre Boulanger

Follow this and additional works at: https://ir.lib.uwo.ca/biophysicspub

Part of the Medical Biophysics Commons

Citation of this paper:

Punithakumar, Kumaradevan; Noga, Michelle; Ben Ayed, Ismail; and Boulanger, Pierre, "Right ventricular segmentation in cardiac MRI with moving mesh correspondences." (2015). Medical Biophysics Publications. 54.

https://ir.lib.uwo.ca/biophysicspub/54 


\title{
Right ventricular segmentation in cardiac MRI with moving mesh correspondences
}

\author{
Kumaradevan Punithakumar ${ }^{\mathrm{a}, \mathrm{b}, *}$, Michelle Noga ${ }^{\mathrm{a}, \mathrm{b}}$, Ismail Ben Ayed ${ }^{\mathrm{c}, \mathrm{d}}$, \\ Pierre Boulanger ${ }^{\mathrm{a}, \mathrm{b}, \mathrm{e}}$ \\ a Servier Virtual Cardiac Centre, Mazankowski Alberta Heart Institute, Edmonton, Alberta, Canada \\ ${ }^{\mathrm{b}}$ Department of Radiology and Diagnostic Imaging, University of Alberta, Edmonton, Alberta, Canada \\ ${ }^{\text {c } G E ~ H e a l t h c a r e, ~ L o n d o n, ~ O n t a r i o, ~ C a n a d a ~}$ \\ d Department of Medical Biophysics, Western University, London, Ontario, Canada \\ e Department of Computing Science, University of Alberta, Edmonton, Alberta, Canada
}

\section{A R T I C L E I N F O}

\section{Article history:}

Received 18 July 2014

Received in revised form

27 November 2014

Accepted 9 January 2015

\section{Keywords:}

Right ventricular function

Segmentation

Moving mesh

Grid generation

Magnetic resonance imaging

Ejection fraction

\begin{abstract}
A B S T R A C T
This study investigates automatic propagation of the right ventricle (RV) endocardial and epicardial boundaries in $4 \mathrm{D}$ (3D+time) magnetic resonance imaging (MRI) sequences. Based on a moving mesh (or grid generation) framework, the proposed algorithm detects the endocardium and epicardium within each cardiac phase via point-to-point correspondences. The proposed method has the following advantages over prior RV segmentation works: (1) it removes the need for a time-consuming, manually built training set; (2) it does not make prior assumptions as to the intensity distributions or shape; (3) it provides a sequence of corresponding points over time, a comprehensive input that can be very useful in cardiac applications other than segmentation, e.g., regional wall motion analysis; and (4) it is more flexible for congenital heart disease where the RV undergoes high variations in shape. Furthermore, the proposed method allows comprehensive RV volumetric analysis over the complete cardiac cycle as well as automatic detections of end-systolic and end-diastolic phases because it provides a segmentation for each time step. Evaluated quantitatively over the 48-subject data set of the MICCAI 2012 RV segmentation challenge, the proposed method yielded an average Dice score of $0.84 \pm 0.11$ for the epicardium and $0.79 \pm 0.17$ for the endocardium. Further, quantitative evaluations of the proposed approach in comparisons to manual contours over 23 infant hypoplastic left heart syndrome patients yielded a Dice score of $0.82 \pm 0.14$, which demonstrates the robustness of the algorithm.
\end{abstract}

(C) 2015 Elsevier Ltd. All rights reserved.

\section{Introduction}

Right ventricular (RV) failure can have numerous aetiologies, which include pulmonary hypertension, cardiomyopathy, myocardial infarction, congenital heart disease, and sepsis [1]. Although clinically evidenced with several studies [2,3], the importance of RV analysis was overlooked in the last decade. Andersen et al. [2] showed that up to $50 \%$ of all regional infarcts are RV related. Cor pulmonale, the enlargement of the RV due to pulmonary hypertension, is the third most common cause of cardiac dysfunction in patients over the age of 50 [3]. RV analysis is much more complicated than LV analysis, and its clinical assessments are more labour-intensive. Both ventricles are composed of multiple layers

\footnotetext{
* Corresponding author at: Department of Radiology and Diagnostic Imaging, University of Alberta, Edmonton, Alberta, Canada. Tel.: +1 7804071871.

E-mail address: punithak@ualberta.ca (K. Punithakumar).
}

that form a 3D network of fibres. However, the shape of the RV is more complex. It generally appears triangular when viewed longitudinally and crescent-shaped when observed from the short-axis view, whereas the LV is ellipsoidal. RV shortening is larger longitudinally than radially [4], and the RV has a wall much thinner than the LV as it ejects blood against approximately $25 \%$ of the LV after-load [5].

Early detection and analysis of the RV dysfunction are helpful in identifying RV dysfunction prior to progression of RV failure. For instance, untreated pulmonary arterial hypertension may evolve rapidly and, therefore, can lead to RV failure and death, with a median survival time of less than 3 years after the diagnosis [6]. The current state-of-the-art solutions in imaging provide an excellent tool for detecting abnormalities in RV function. Magnetic Resonance Imaging (MRI) is considered as the current gold standard for non-invasive anatomical and functional assessment of the RV; it allows detailed RV evaluation [1]. Cardiac MRI is used to evaluate the right ventricle in a number of congenital heart conditions 
[7] and in pulmonary artery hypertension [8]. MRI has several important advantages over echocardiography. These include excellent image quality, lack of geometric assumptions, no endocardial dropout, and no foreshortening of the apex. Clinical measurements such as the RV ejection fraction (RVEF) and volumes have important diagnostic, prognostic and therapeutic implications for patients with acquired heart disease, particularly those who need cardiac function follow-up $[9,10]$. For quantitative functional analysis, and to obtain clinical measurements such as volume or RVEF, it is essential to delineate the RV. Manual delineation of the RV boundaries in all magnetic resonance (MR) images ${ }^{1}$ is tedious and time-consuming. Therefore, it is a common clinical practice to delineate the RV boundaries only in end diastolic and end systolic phases. Automating the process has bestirred a significant research attention recently, and has been the subject of a Medical Image Computing and Computer Assisted Intervention (MICCAI) 2012 RV Segmentation Challenge (RVSC) [11-19].

Due to its complex morphology and function, RV segmentation in 3D+time is acknowledged more challenging than LV segmentation $[4,20]$. Most of the research efforts in the last decade focused on the LV [21-27]. RV segmentation remains an unsolved problem, with difficulties arising from the thin and ill-defined borders of the RV, its crescent-shaped structure and its complex deformations over time. Another problem associated with RV border detection is the presence of adjacent epicardial fat, which blurs the anterior margins of the RV [4]. Furthermore, RV segmentation methods need to consider the intensity similarities between different connected cardiac regions. For instance, the papillary muscles and heart wall have approximately the same intensity profiles. Therefore, standard segmentation methods based solely on intensity information cannot yield accurate results. To overcome these difficulties, most of the existing methods use either atlasbased techniques $[11,15,18,28-30]$ or prior geometric properties $[12,17,31-33]$, e.g., the shape of the RV is learned a priori from a finite training set. The main drawbacks of shape or atlas based approaches are (1) the requirement for large, manually segmented training sets; and (2) the high dependence of the results on the specific choice of a training set, which can lead to biases towards a particular cardiac pathology or towards the properties of normal subjects, e.g., the mean RV shape within the training data. The shape of the RV and its position relative to the LV may vary significantly from one subject to another (Refer to Fig. 1 for a typical RV and the $\mathrm{RV}$ of a hypoplastic left heart syndrome patient (HLHS)). HLHS is a congenital heart malformation, where the structures of the left side of the heart are underdeveloped or too small. The initial surgical palliation causes the RV to pump blood to the entire body and the lungs. Assessing the RV in MRI prior to further surgery is important but is difficult in the case of patients that are young infants. Methods that rely on the spatial correspondences between the LV and RV may not be accurate for diseased hearts because such correspondences may vary significantly. For example, the method in [34] assumes that the RV is to the left of, and generally above, the LV. This assumption may not be true for HLHS patients such as the one depicted in Fig. 1(b), where the LV is very small and the RV occupies a position different than usual.

The shape of the RV might be considerably different at endsystole in comparison to end-diastole. Therefore, in general, shape-based approaches have difficulty in capturing the RV at endsystole. The results published by the recent RVSC at the MICCAI 2012 conference confirm this. These results show that most of the existing methods have the highest segmentation error at endsystole $[11-15,17,18]$. The best reported Dice Metric (DM) values

\footnotetext{
1 Typically, the number of images per subject is equal to 200 .
}

among the seven participants were 0.72 and 0.77 for endocardium and epicardium, respectively. Due to its smaller size, inaccuracies in the segmentation of the RV at end-systole affect the value of clinical measurements such as the RVEF.

To tackle the problem of $4 \mathrm{D}(3 \mathrm{D}+$ time $)$ data sets, we propose a semi-automatic approach based on a moving mesh (or grid generation) framework. Given a user-provided segmentation of a single frame in a cardiac sequence, the proposed method segments both endocardial and epicardial borders of the RV via point-to-point correspondences (Refer to Fig. 3). The proposed method allows comprehensive RV volumetric analysis over the complete cardiac cycle as well as automatic detections of end-systolic and enddiastolic phases because it provides segmentation for each time step.

Our method is related to propagation/registration frameworks, which have been used previously for tracking the left ventricular boundaries. An example is the work in [35,36], which optimises a sum of energy terms consisting of a squared $L_{2}$ norm and a Sobolev smoothing term. Based on the concept of equivalent volume elements of a compact Riemannian manifold [37] and yielding a unique solution by solving an div-curl system, the proposed moving mesh approach is fundamentally different from $[35,36]$ : The proposed moving mesh prevents mesh folding, i.e., grid lines of the same grid family will not cross each other, an essential attribute in tracking cardiac tissues from a sequence of images. Furthermore, it allows setting explicitly the minimum and maximum values for the compressibility of the mesh, which can be used to imitate cardiac tissue deformation, thereby yielding a better point correspondence mapping.

A preliminary conference version of this work appeared in IEEE EMBC 2013 [38]. This journal version expands on [38] with:

(1) A wider experimental investigation that includes more patient data and statistical validations. Additional experimental evaluations include: (a) 16 new subjects bringing the total adult subjects to 48; and (b) 23 infant HLHS patients.

(2) A much broader, more informative/rigorous discussion of the subject. This includes an algorithmic description of the moving mesh generation and a more detailed discussion on the challenges in segmenting the RV under abnormal conditions such as HLHS.

The remainder of this paper is organized as follows. Section 2 describes the moving mesh framework used in the study. Experimental evaluations over RVSC and HLHS data sets as well as comparisons of the proposed method with other recent methods on RVSC data sets are described in Section 3. Section 4 provides a discussion and the limitations of the proposed study. Finally, conclusions are given in Section 5.

\section{Methods}

We propose to use a 2D moving mesh (or grid generation) framework [37] to compute point-to-point correspondences between the $k$ th image $T_{k}$ and $(k+1)$ th image $T_{k+1}$ (for $k=1, \ldots, K-1$ ) defined over $\Omega \subset \mathbb{R}^{2}$ ( $K$ is the total number of frames in a cardiac cycle), thereby obtaining a sequence of points over time. Fig. 2 depicts the flow diagram for computing such point-to-point correspondences. We state the problem as an optimisation of a similarity/dissimilarity measure [39]:

$\hat{\phi}=\operatorname{argopt} E_{s}\left(T_{k}, T_{k+1}, \phi(\xi)\right)$

where $\xi \in \Omega$ denotes pixel location, $\phi: \Omega \rightarrow \Omega$ a transformation function and $E_{S}(\cdot)$ a measure of dissimilarity. We will use the squared $L_{2}$ norm as the dissimilarity measure $E_{S}(\cdot)$ [40]. As this 


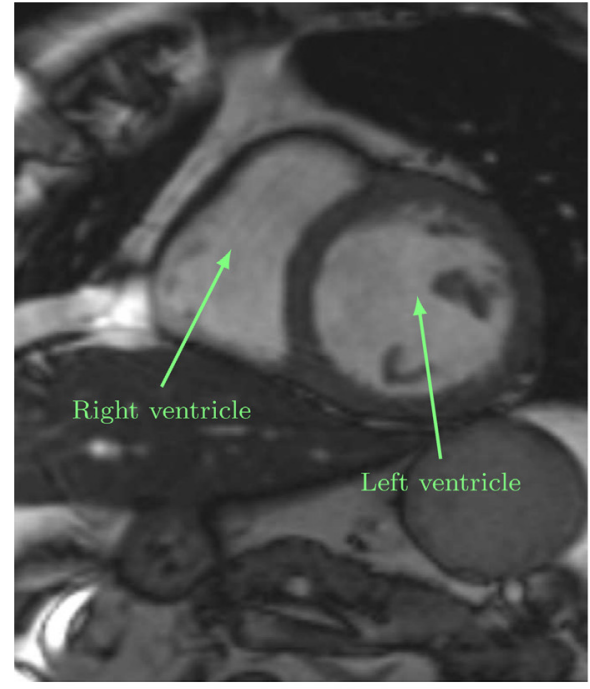

(a) Typical

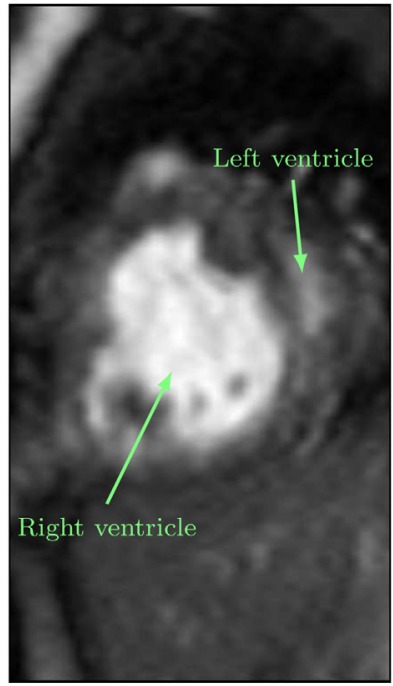

(b) Hypoplastic left heart syndrome

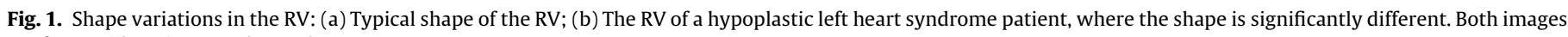
are from mid-cavity at end-systole.

problem may not have a unique solution and require more constraints, we introduce in the following a deformation field using a monitor function $\mu$ and curl of end velocity field $\gamma$, where $\mu: \Omega \rightarrow$ $\mathbb{R}$ and $\gamma: \Omega \rightarrow \mathbb{R}$.

\subsection{Moving mesh generation}

Let $\mu(\xi)$ be a continuous monitor function constrained by:

$\int_{\Omega} \mu=|\Omega|$.

The purpose of this step is to find a transformation $\phi: \Omega \rightarrow \Omega$, $\partial \Omega \rightarrow \partial \Omega$, so that

$J_{\phi}(\xi)=\mu(\xi)$ where $J_{\phi}$ is the Jacobian determinant of the transformation. The following computations yield a transformation $\phi$, which verifies (3).

Step 1: Compute a vector field $\rho(\xi)$, defined by $\operatorname{div} \rho(\xi)=\mu(\xi)-1$.

Step 2: Build a velocity vector field from $\rho(\xi)$ :

$v_{t}(\xi)=\frac{\rho(\xi)}{t+(1-t) \mu(\xi)}, \quad t \in[0,1]$,

where $t$ is an artificially introduced (algorithmic) time.

Step 3: Finally, $\phi$ is obtained by solving the following ODE:

$\frac{d \psi(\xi, t)}{d t}=v_{t}(\psi(\xi, t)), \quad t \in[0,1], \psi(\xi, t=0)=\xi$,

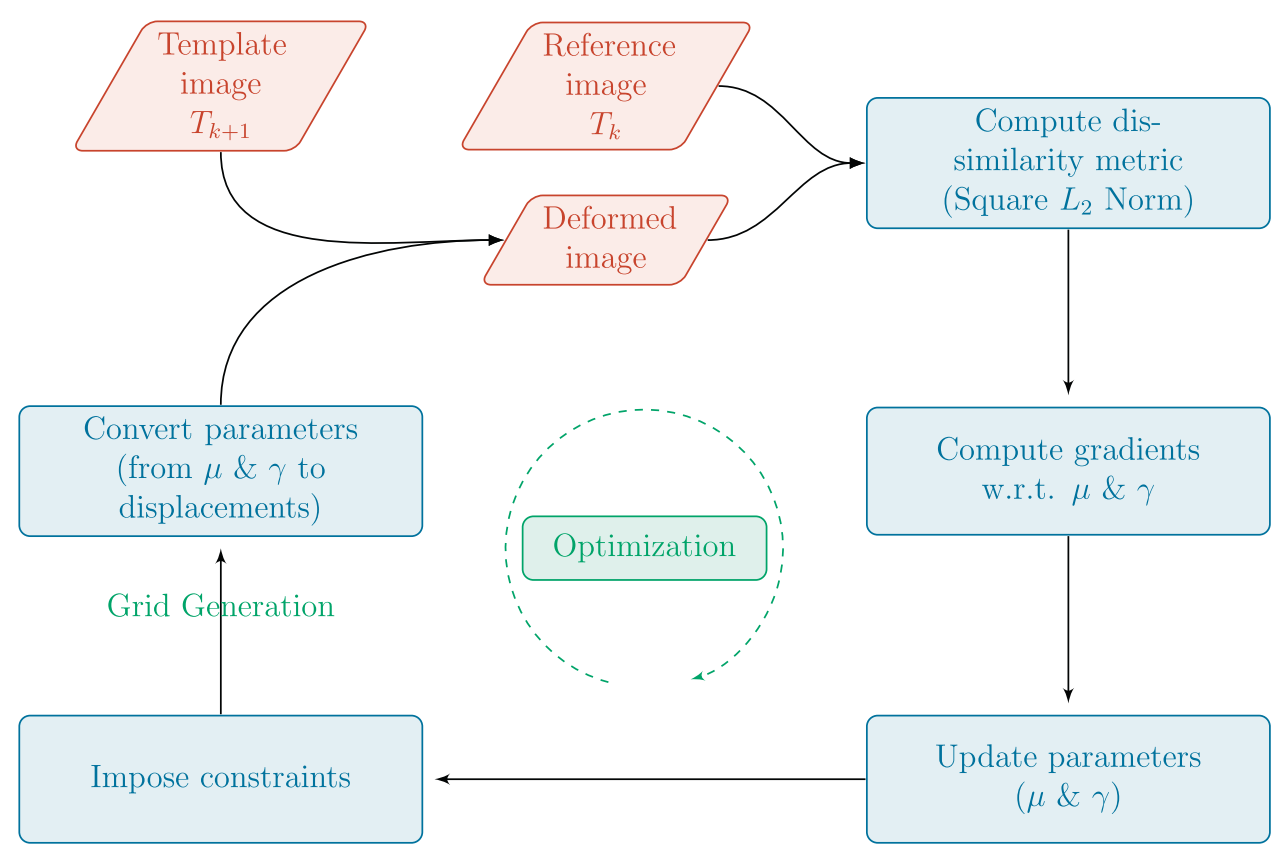

Fig. 2. Computation of moving mesh correspondences. 


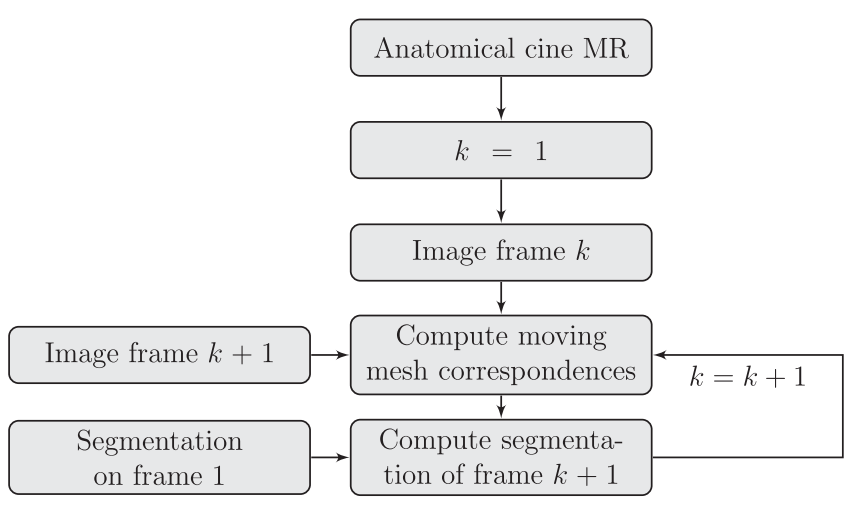

Fig. 3. The proposed approach to delineate endocardium and epicardium from a sequence of cine MR images using moving mesh correspondences.

The above problem may have multiple solutions. We solve the div-curl system under the Dirichlet boundary condition by adding to (4) a constraint on the curl of $\rho(\xi)$, which guarantees obtaining a unique solution:

$\left\{\begin{array}{l}\operatorname{div} \rho(\xi)=\mu(\xi)-1 \\ \operatorname{curl} \rho(\xi)=\gamma(\xi)\end{array}\right.$

with null boundary condition $\rho(\xi)=0 \forall \xi \in \partial \Omega$, where $\gamma(\xi)$ is a continuous function over $\Omega$. Hence, the transformation can be fully parameterized by $J_{\phi}(\xi)$ and $\gamma(\xi)$. We ensure the uniqueness of the solution using the Dirichlet boundary condition [41]. The transformation function $\mu(\cdot)$ and curl $\gamma(\cdot)$ correspond to the radial and rotational components of the deformation field, respectively. The Dirichlet boundary conditions may cause the motion errors to be higher at the image boundaries and, therefore, we pad both images by zeros.

With the above parametrization, we reformulate (1) as the following constrained optimization problem:

Problem: Given two images $T_{k}$ and $T_{k+1}$, defined over $\Omega$, find a function pair $\{\mu(\xi), \gamma(\xi)\}$ that optimizes cost (1) s.t.:

$$
\left\{\begin{array}{l}
\int_{\Omega} \mu(\xi) d \xi=|\Omega| \\
\tau_{h}>\mu(\xi)>\tau_{l}, \quad \xi \in \Omega^{\prime} \subset \Omega
\end{array}\right.
$$

where $0<\tau_{l}$ ensuring that $\phi_{\mu, \gamma}$ is a diffeomorphism, and $\Omega^{\prime}$ is a subregion of image domain $\Omega$.

Constraint (8a) ensures that the areas of the domain and codomain are equal after transformation, and constraint (8b) limits the amount of compressibility, which is controlled by parameters $\tau_{l}$ and $\tau_{h}$, within sub-region $\Omega^{\prime}$. Note that a diffeomorphism corresponds to a positive transformation Jacobian determinant, which is enforced explicitly via the monitor function [37].

The above problem can be solved by a step-then-correct optimization strategy as described in Algorithm 1. We compute a sequence of corresponding points on endocardial as well as epicardial borders in all the frames of a cardiac sequence using transformation function $\hat{\phi}$, given the segmentation on the first frame. For current frame $T_{k+1}$, the amount of deformation with respect to previous neighbouring frame $T_{k}$ is smaller than the deformations with respect to other frames that occur further in the temporal domain, e.g., first frame $T_{1}$. Therefore, in computing the transfer function, we set the study image to previous frame $T_{k}$ rather than first frame $T_{1}$. This improves accuracy and convergence time, and encourages consistency of the solutions over time. Fig. 3 shows the steps of computing the contours for all the frames of a cardiac cycle.

Algorithm 1. Step-then-correct optimization

Given image pair $T_{1}$ and $T_{k}$, consider the following steps.

Step 1 Compute the gradients of $\mu$ and $\gamma$ which we denote respectively by $\nabla \mu\left(T_{1}, T_{k}, \phi\right)$ and $\nabla \gamma\left(T_{1}, T_{k}, \phi\right)$

Step 2 Terminate if step size $\delta<\delta_{\text {th }}$ or a maximum number of iterations is reached; otherwise, update $(\mu, \gamma)$ by ( $i$ is the iteration number):

$$
\mu^{i+1}=\mu^{i}+\delta \frac{\nabla \mu E_{S}}{\max \left|\nabla \mu E_{S}\right|} \quad \text { and } \quad \gamma^{i+1}=\gamma^{i}+\delta \frac{\nabla \gamma E_{S}}{\max \left|\nabla \gamma E_{S}\right|}
$$

Step 3 For each pixel location $\xi \in \Omega^{\prime} \subset \Omega$, impose constraint (8b) by

$$
\mu^{i+1}(\xi) \leftarrow \max \left(\mu^{i+1}(\xi), \tau_{l}\right) \text { and } \mu^{i+1}(\xi) \leftarrow \min \left(\mu^{i+1}(\xi), \tau_{h}\right)
$$

For each pixel location $\xi \in \Omega$, impose constraint (8a) by

$$
\mu^{i+1}(\xi) \leftarrow \mu^{i+1}(\xi) \frac{|\Omega|}{\sum_{\xi \in \Omega} \mu^{i+1}(\xi)}
$$

Step 4 Find a vector field $\rho(\xi)$ which satisfies:

$$
\left\{\begin{array}{l}
\operatorname{div} \rho(\xi)=\mu^{i+1}(\xi)-1 \\
\operatorname{curl} \rho(\xi)=\gamma^{i+1}(\xi)
\end{array}\right.
$$

with null boundary condition $\rho(\xi)=0, \forall \xi \in \partial \Omega$. Compute the transformation $\phi(\xi)=\psi(\xi, t=1)$ by finding the solution to the ordinary differential equation

$$
\frac{d \psi(\xi, t)}{d t}=v_{t}(\psi(\xi, t)) \quad t \in[0,1]
$$

with $\psi(\xi, t=0)=\xi$. The velocity vector field $v_{t}$ is given by

$$
v_{t}(\xi)=\frac{\rho(\xi)}{t+(1-t) \mu^{i+1}(\xi)} \quad t \in[0,1]
$$

Step 5 Compute cost $E_{S}$. If it improves, $i \leftarrow i+1$, go to Step 1; otherwise, decrease $\delta$ and go to Step 2 .

\section{Experimental results}

The proposed method was evaluated over a data set composed of 48 subjects provided by the RVSC, MICCAI 2012 [16] and a data set composed of 23 Hypoplastic Left Heart Syndrome (HLHS) patients. For the moving mesh, grid sampling was set equal to the pixel spacing. The size of the grid was selected automatically based on a bounding box containing the initial segmentation drawn on the first frame. A margin of 5 pixels around the bounding box was added to allow deformations outside the bounding box. For the step-thencorrect algorithm, we set $\delta_{t h}=0.02$ and the maximum number of iterations to 30. The initial value of $\delta$ and the factor to reduce $\delta$ were set to 0.5 and $2 / 3$, respectively. Given the high variability in right 
Table 1

Details of the RVSC datasets used in evaluation of the proposed method.

\begin{tabular}{ll}
\hline Description & Value \\
\hline Number of subjects & 48 \\
Patient age & $50.8 \pm 17.6$ years \\
Patient age range & $17-88$ years \\
Sex, $\mathrm{m} / \mathrm{f}$ & $32 / 16$ \\
Short-axis image size & $(256 \times 216)$ or $(216 \times 256)$ pixels \\
Number of frames $(K)$ & 20 \\
Heart rate & $55-106 \mathrm{bpm}$ \\
Pixel spacing & $(0.57 \times 0.57)-(0.97 \times 0.97) \mathrm{mm}$ \\
\hline
\end{tabular}

ventricular motion, the following parameter values were used for all cases so to allow large tissue deformations: $\tau_{h}=4$ and $\tau_{l}=0.25$.

For quantitative analysis, two criteria were used to evaluate the similarities between the manual and automatic segmentations:

The Dice Metric (DM). We computed the DM, a common measure of similarity between manual and automatic segmentations. The DM is given by

$D M\left(V_{a}, V_{m}\right)=\frac{2 V_{a m}}{V_{a}+V_{m}}$,

where $V_{a}, V_{m}$ and $V_{a m}$ are, respectively, the volumes of the automatically segmented region, the corresponding manually delineated region, and the intersection between them. Note that DM is always between 0 and 1 , where 1 means a perfect match.

The Hausdorff Distance (HD). We computed the HD [42], a symmetric measure of distance between both automatic and manual contours. Let us denote automatic and manual contours by $C_{a}$ and $C_{m}$, respectively. For each point $p_{a}^{i}$ on $C_{a}$, we compute the distances to all the points $p_{m}^{j}$ on $C_{m}$. The HD is given by

$H D\left(C_{a}, C_{m}\right)=\max \left(\max _{i}\left(\min _{j}\left(d\left(p_{a}^{i}, p_{m}^{j}\right)\right)\right), \max _{j}\left(\min _{i}\left(d\left(p_{a}^{i}, p_{m}^{j}\right)\right)\right)\right)$

where $d(\cdot)$ is the Euclidean distance. The HD is computed in mm, with the spatial resolution obtained from the pixel spacing in the DICOM header.

\subsection{RV segmentation challenge data}

The proposed method was evaluated over the training and testing sets provided by the RVSC [16]. Each data set consists of short-axis MRI volumes of 16 subjects. The data sets were acquired on 1.5T MRI scanners (Symphony Tim, Siemens Medical Systems, Erlangen, Germany) with steady-state free precession acquisition mode. Sequence parameters were as follows: $\mathrm{TR}=50 \mathrm{~ms} ; \mathrm{TE}=1.7 \mathrm{~ms}$; flip angle $=55$; slice thickness $=7 \mathrm{~mm}$; matrix size $=256 \times 216$; Field of view $=360-420 \mathrm{~mm}$; 20 images per cardiac cycle. The details of the data sets are presented in Table 1. The data was acquired from June 2008 to August 2008 at the Rouen University Hospital, Rouen, France and all patients gave written informed consent. Participants in the study had the following indications for MRI: myocarditis, ischaemic cardiomyopathy, suspicion of arrhythmogenic right ventricular dysplasia, dilated cardiomyopathy, hypertrophic cardiomyopathy, aortic stenosis. More details about the data can be found at the RVSC website. ${ }^{2}$ Ground truth manual segmentations were provided only for the training set. For testing data sets, initial manual contours were drawn using a programme developed in-house that uses spline smoothing, and therefore, require a small number of points to be provided by the user. In order to assess the performance of the proposed algorithm on the testing sets, we submitted the automatic contours to the

\footnotetext{
2 http://www.litislab.eu/rvsc/.
}

Table 2

Mean and standard deviation of Dice Metric (DM) and Hausdorff Distance (HD) between the obtained segmentations and manual delineations at end-systole.

\begin{tabular}{lll}
\hline & $\mathrm{DM}$ & $\mathrm{HD}(\mathrm{mm})$ \\
\hline Training set & & \\
Endocardium & $0.82(0.15)$ & $7.07(4.03)$ \\
Epicardium & $0.86(0.10)$ & $7.53(3.73)$ \\
\hline
\end{tabular}

RVSC organizers, who in return, provided us with the performance measures.

\subsubsection{Statistical performance evaluation}

Table 2 reports the DM and HD values for the Training set at ES. Table 3 reports the DM and HD values for the Test 1 set for the proposed approach and other methods from the MICCAI 2012 RVSC evaluated at the end-systole. The testing-set evaluations were performed by the challenge organizers. The higher the DM or the lower the HD, the better the performance. Two of the competing methods did not report epicardial segmentations and are denoted as N/A. The table shows that our method performed significantly better than the other methods for both endocardial and epicardial segmentations. Evaluated over the full data set (i.e., 48 subjects from the training and testing sets), the proposed method yielded average Dice scores of 0.79 and 0.84 , respectively, for endocardial and epicardial segmentations.

We further evaluated the performance of the proposed method for estimating clinical measurements such as RV end-diastolic volume (RVEDV), RV end-systolic volume (RVESV) and RVEF. Fig. 4 reports the Bland-Altman analysis for RVEDV, RVESV, RVEF and right ventricular mass (RVM). In the analysis, two parameters were computed: (1) bias was calculated by the mean difference between the automatic and manual measurements; and (2) the limits of agreement (LOA) was calculated by 1.96 standard deviations of the differences and covers the range of values which includes $95 \%$ of all the differences between the automatic and manual measurements. Fig. 4(a) and (d) correspond to the manual contours on the initial frame, where manual contour 1 was drawn by the RVSC organizers and manual contour 2 was drawn by us. We excluded the training set in the analysis of RVEDV and RVM, since the manual contours were provided by the RVSC organizers and we did not draw contours on the initial frame. Fig. 4(b) and (c) correspond to the auto contours by the proposed method and the manual contours provided by the RVSC organizers. The bias and LOA values for RVEDV, RVESV, RVEF and RVM were 5.4 $( \pm 21.0) \mathrm{ml}, 7.1( \pm 17.9) \mathrm{ml}$, $-0.04( \pm 0.09)$ and $7.3( \pm 14.5)$, respectively.

Table 4 reports the overall results for endocardial segmentation over end-systolic and end-diastolic phases. Only anonymized results for RSVC participants are available since individual results for Test2 set are not publicly available [34]. Table 5 reports the overall results for epicardial segmentation. The overall values for our method in Tables 4 and 5 include the manual segmentation at end-diastole. The results show that the proposed method yielded the best performance in comparison to other related methods.

\subsubsection{Visual inspection}

In Fig. 5, we give representative examples of segmented endocardial and epicardial borders of the RV over a complete cardiac cycle. These examples show that the proposed method accurately included the papillary muscles inside the target cavity, although these have intensity profiles similar to the RV myocardium. We could also see that the contours closely follow the RV wall even though parts of the borders are not visible in many frames.

The MATLAB parallel implementation of our algorithm running on a MacPro with dual 6-core Intel Xeon processors took $4.7 \pm 1.7 \mathrm{~s}$ 
Table 3

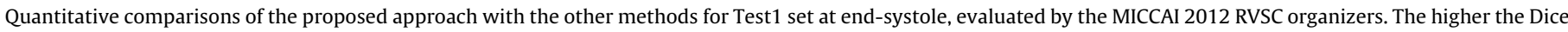
Metric (DM) or the lower the Hausdorff Distance (HD), the better the performance.

\begin{tabular}{|c|c|c|c|c|}
\hline \multirow[t]{2}{*}{ Method } & \multicolumn{2}{|c|}{ Endocardium } & \multicolumn{2}{|l|}{ Epicardium } \\
\hline & DM & $H D$ & DM & HD \\
\hline \multicolumn{5}{|l|}{ Semi-automatic } \\
\hline Our method & $0.77(0.16)$ & $9.64(4.15)$ & $0.82(0.10)$ & $9.99(3.85)$ \\
\hline Bai et al. [11] & $0.69(0.25)$ & $11.16(5.53)$ & $0.77(0.17)$ & $11.72(5.44)$ \\
\hline Grosgeorge et al. [12] & $0.69(0.23)$ & $10.56(5.54)$ & $0.78(0.15)$ & $11.09(5.34)$ \\
\hline Maier et al. [13] & $0.69(0.02)$ & $14.75(0.40)$ & $\mathrm{N} / \mathrm{A}$ & $\mathrm{N} / \mathrm{A}$ \\
\hline Nambakhsh et al. [14] & $0.48(0.25)$ & $23.19(9.71)$ & $\mathrm{N} / \mathrm{A}$ & $\mathrm{N} / \mathrm{A}$ \\
\hline \multicolumn{5}{|l|}{ Automatic } \\
\hline Ou et al. [15] & $0.53(0.32)$ & $20.44(17.80)$ & $0.60(0.30)$ & $21.91(18.92)$ \\
\hline Ringenberg et al.[34] & $0.77(0.18)$ & $10.71(7.69)$ & $0.82(0.13)$ & $11.52(7.70)$ \\
\hline Wang et al. [17] & $0.50(0.34)$ & 27.99 (24.97) & $0.55(0.36)$ & $27.58(24.82)$ \\
\hline Zuluaga et al. [18] & $0.72(0.27)$ & $11.41(10.49)$ & $0.77(0.23)$ & $11.81(9.46)$ \\
\hline
\end{tabular}

to process a sequence of 19 image slices for both endocardial and epicardial borders.

\subsection{Hypoplastic Left Heart Syndrome patients}

The proposed method was evaluated over 23 Hypoplastic Left Heart Syndrome (HLHS) patients. The data sets were acquired on 1.5T MR scanners (Avanto and Sonata; Siemens Healthcare, Erlangen, Germany). The protocol was approved by the University of Alberta Health Research Ethics Board. The average age of subjects is $0.45 \pm 0.30$ years. The minimum and maximum ages of subjects

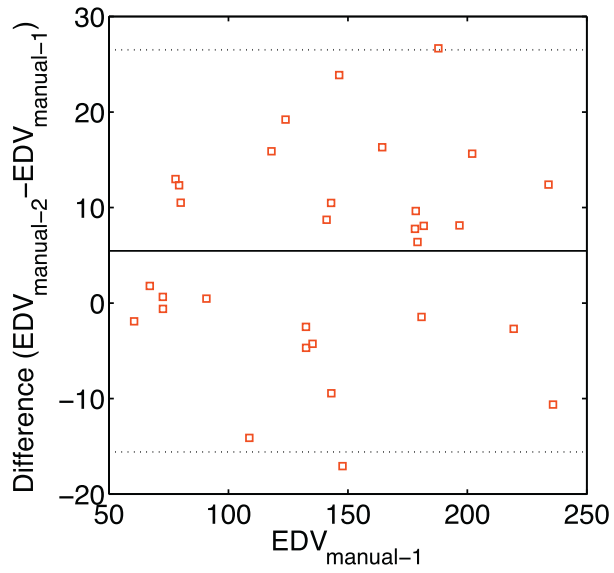

(a) RVEDV

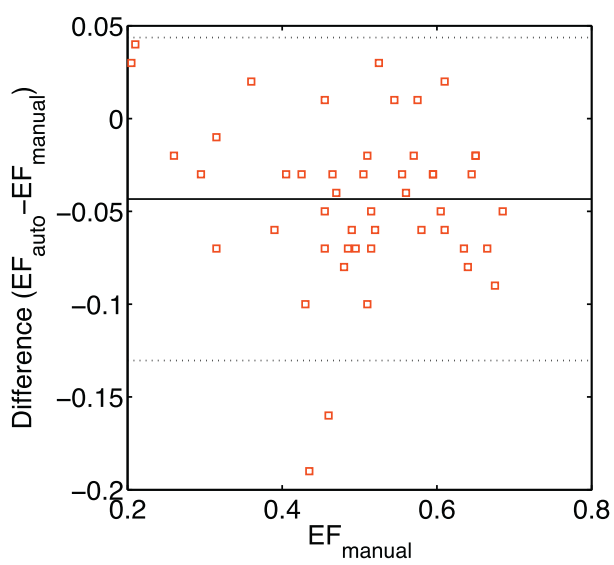

(c) RVEF are 0.12 and 1.39 years, respectively. The details of the datasets are presented in Table 6 . The ground truth manual delineations were performed by an experienced radiologist using a commercial software (Argus, Siemens).

In Fig. 6, we give representative examples showing RV deformations and endocardial segmentations computed using the proposed method. As depicted in the figure, the shape of the $\mathrm{RV}$ of the HLHS patients is significantly different from a normal RV. The example demonstrates that our method yielded accurate tracking of the boundary despite the variability in shape.

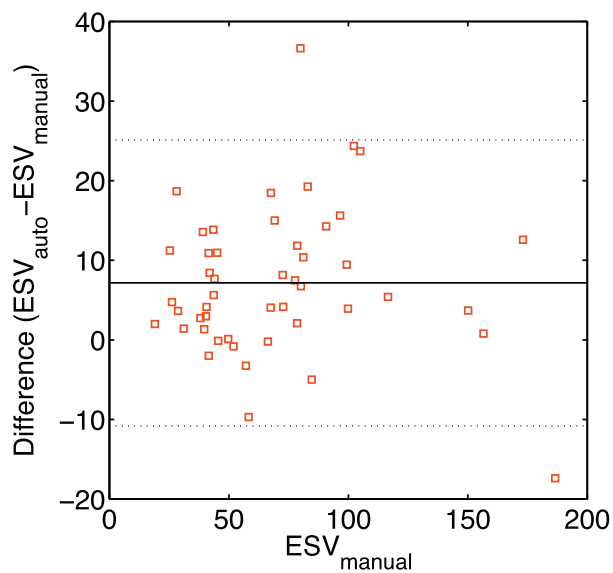

(b) RVESV

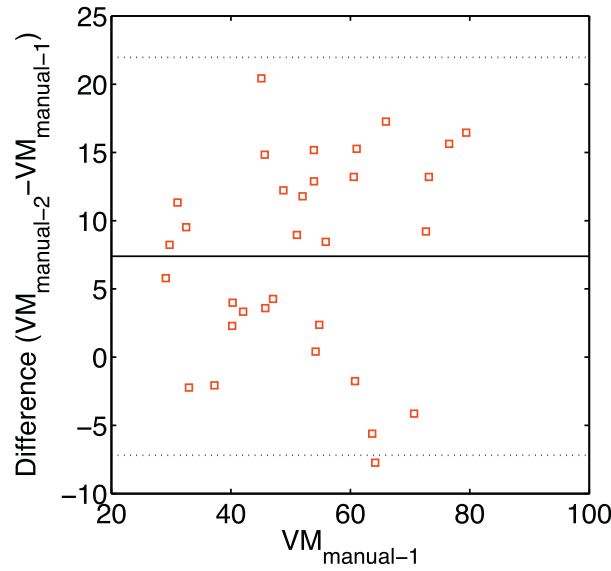

(d) RVM

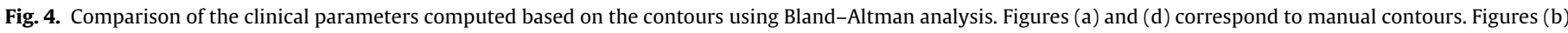
and (c) correspond to the auto contours. 
Table 4

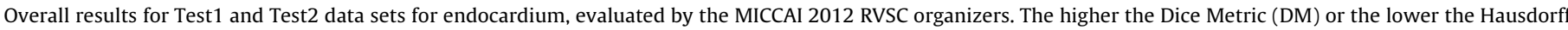
Distance (HD), the better the performance.

\begin{tabular}{|c|c|c|c|c|}
\hline \multirow[t]{2}{*}{ Method } & \multicolumn{2}{|l|}{ Test1 } & \multicolumn{2}{|l|}{ Test2 } \\
\hline & DM & HD & $\mathrm{DM}$ & HD \\
\hline Our method $^{\mathrm{a}}$ & $0.83(0.13)$ & $7.72(3.97)$ & $0.85(0.15)$ & $6.49(4.44)$ \\
\hline Ringenberg et al. [34] & $0.83(0.16)$ & $9.05(6.98)$ & $0.83(0.18)$ & $8.73(7.62)$ \\
\hline Team 1 & $0.76(0.20)$ & $9.97(5.49)$ & $0.81(0.16)$ & $7.28(3.58)$ \\
\hline Team 2 & $0.57(0.33)$ & $28.44(23.57)$ & $0.61(0.34)$ & $22.20(21.74)$ \\
\hline Team 3 & $0.78(0.23)$ & $10.51(9.17)$ & $0.73(0.27)$ & $12.50(10.95)$ \\
\hline Team 4 & $0.55(0.32)$ & $23.16(19.86)$ & $0.61(0.29)$ & $15.08(8.91)$ \\
\hline Team 5 & $0.78(0.20)$ & $9.26(4.93)$ & $0.76(0.23)$ & $9.77(5.59)$ \\
\hline Team 6 & $0.59(0.24)$ & $20.21(9.72)$ & $0.56(0.24)$ & $22.21(9.69)$ \\
\hline Team 7 & $0.80(0.19)$ & $11.15(6.62)$ & $0.77(0.24)$ & $9.79(5.38)$ \\
\hline
\end{tabular}

a The overall values include the manual segmentation at end-diastole.

Manual

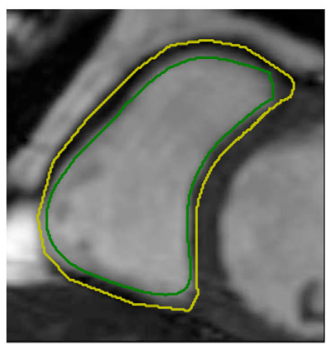

Frame 6

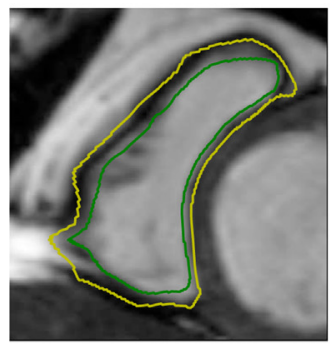

Frame 11

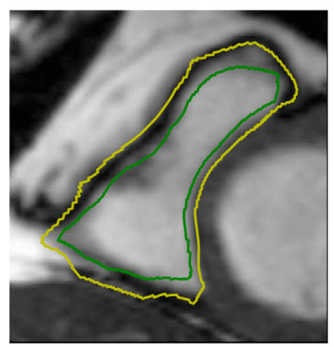

Frame 16

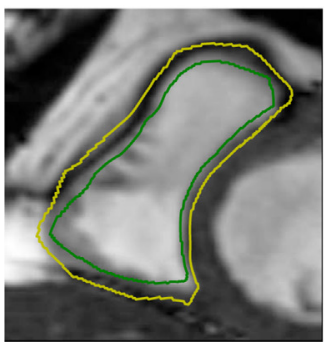

Frame 2

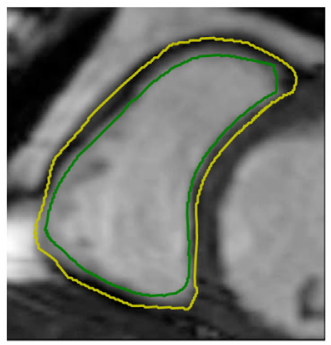

Frame 7

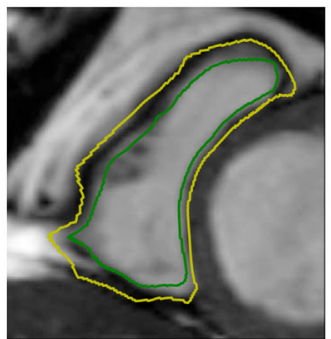

Frame 12

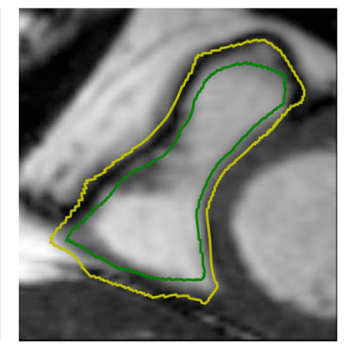

Frame 17

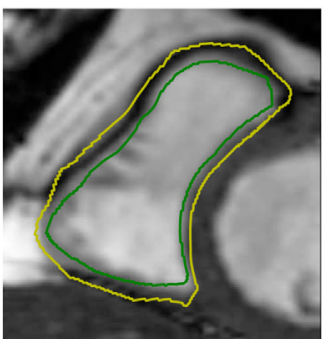

Frame 3

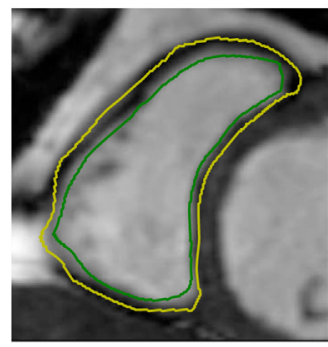

Frame 8

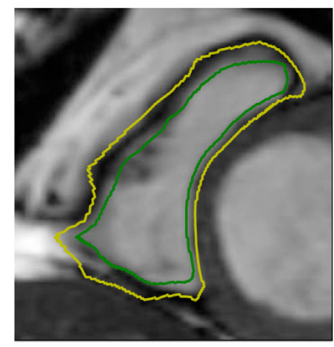

Frame 13

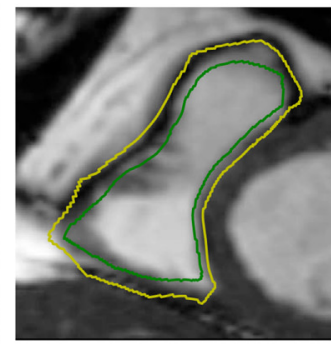

Frame 18

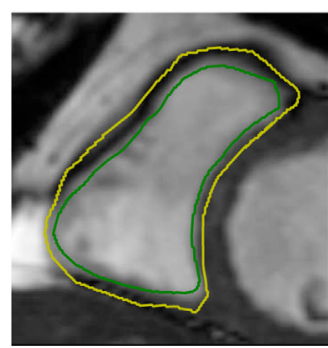

Frame 4

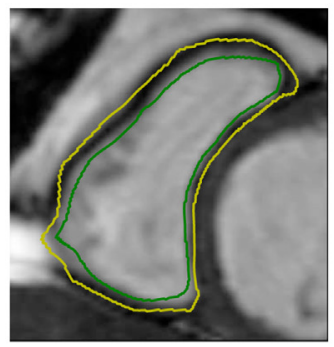

Frame 9

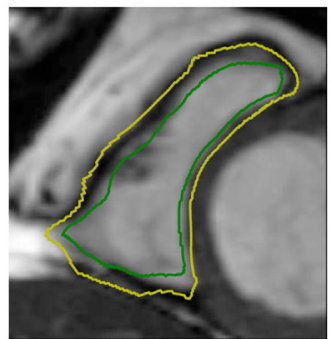

Frame 14

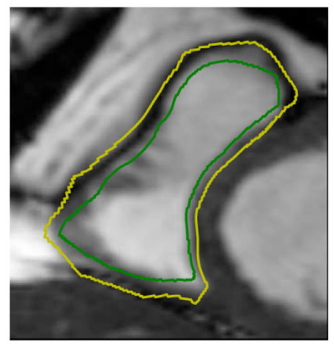

Frame 19

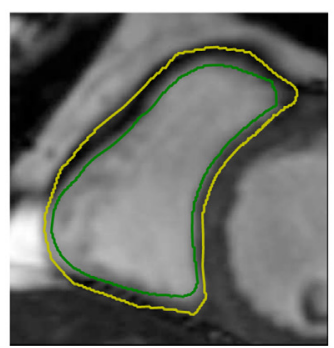

Frame 5

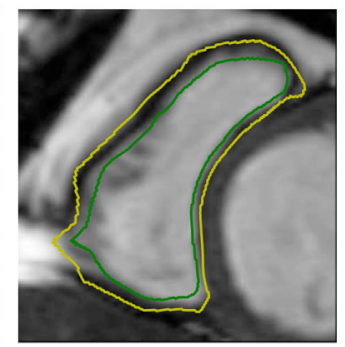

Frame 10

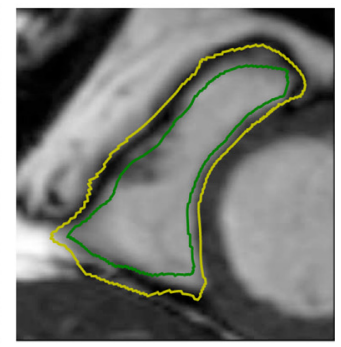

Frame 15

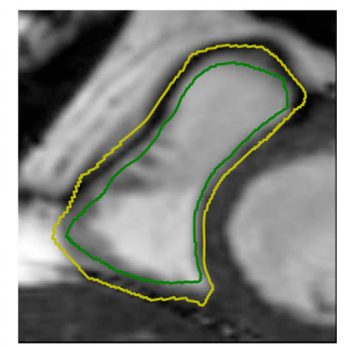

Frame 20

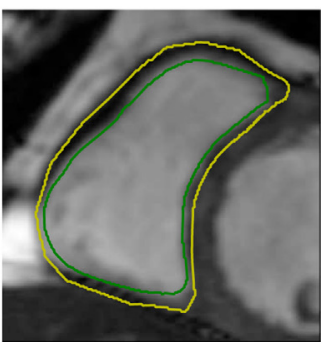

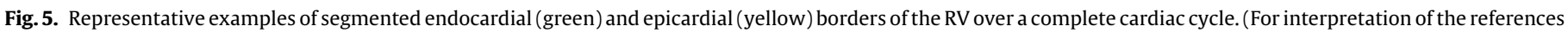
to color in this figure legend, the reader is referred to the web version of the article.) 
Table 5

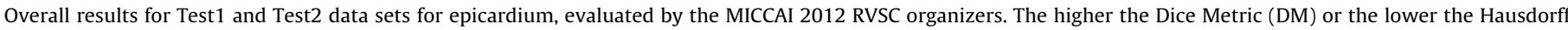
Distance (HD), the better the performance.

\begin{tabular}{|c|c|c|c|c|}
\hline \multirow[t]{2}{*}{ Method } & \multicolumn{2}{|l|}{ Test1 } & \multicolumn{2}{|l|}{ Test2 } \\
\hline & DM & HD & $\mathrm{DM}$ & HD \\
\hline Our method ${ }^{\mathrm{a}}$ & $0.87(0.08)$ & $8.08(3.80)$ & $0.88(0.10)$ & $6.95(3.98)$ \\
\hline Ringenberg et al. [34] & $0.86(0.11)$ & $9.60(7.01)$ & $0.86(0.14)$ & $9.00(7.46)$ \\
\hline Team 1 & $0.82(0.13)$ & $10.40(5.45)$ & $0.85(0.11)$ & $8.32(3.70)$ \\
\hline Team 2 & $0.62(0.35)$ & $26.71(22.90)$ & $0.64(0.35)$ & $22.14(21.61)$ \\
\hline Team 3 & $0.82(0.19)$ & $10.94(8.32)$ & $0.77(0.24)$ & $12.70(10.44)$ \\
\hline Team 4 & $0.58(0.29)$ & $22.53(18.06)$ & $0.68(0.25)$ & $15.17(8.88)$ \\
\hline Team 5 & $0.83(0.14)$ & $9.64(4.95)$ & $0.80(0.18)$ & $10.34(5.41)$ \\
\hline
\end{tabular}

a The overall values include the manual segmentation at end-diastole.

Manual

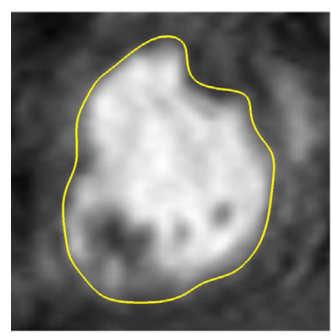

Frame 11

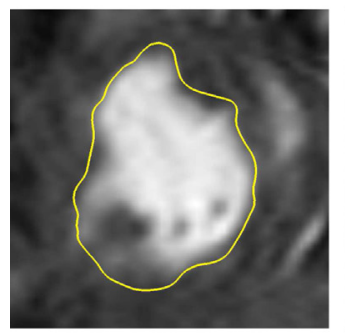

Frame 21

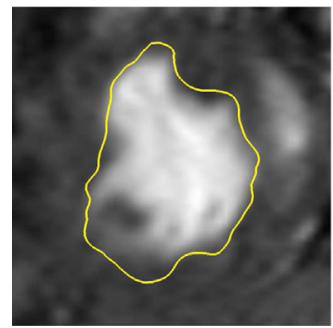

Frame 3

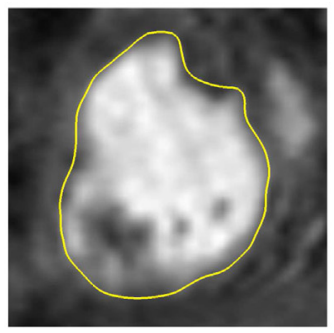

Frame 13

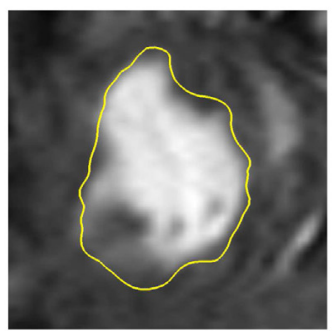

Frame 23

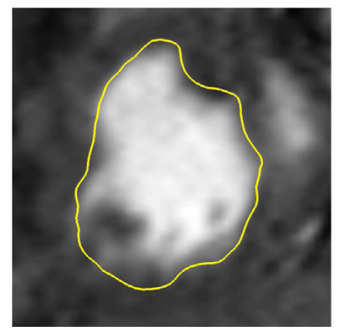

Frame 5

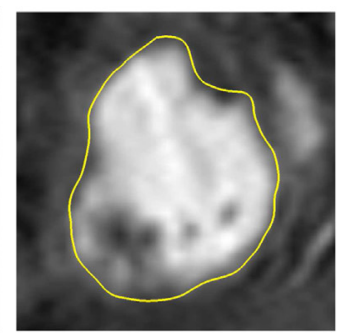

Frame 15

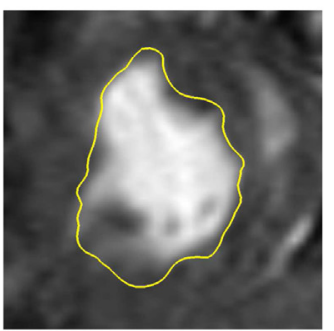

Frame 25

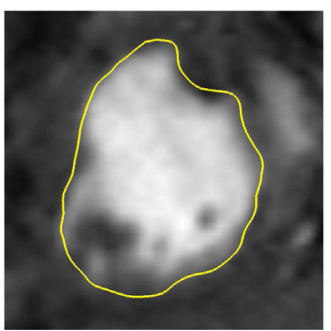

Frame 7

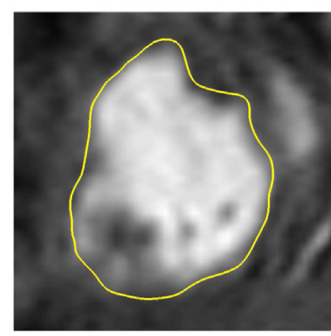

Frame 17

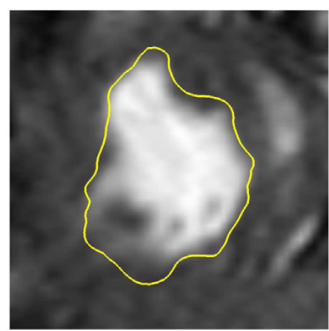

Frame 27

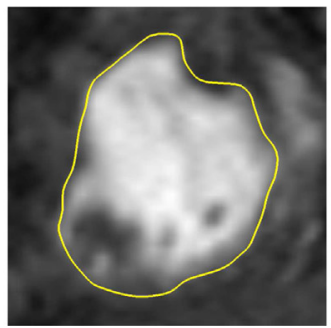

Frame 9

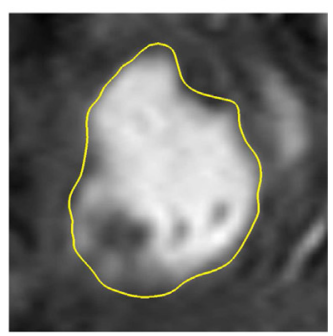

Frame 19

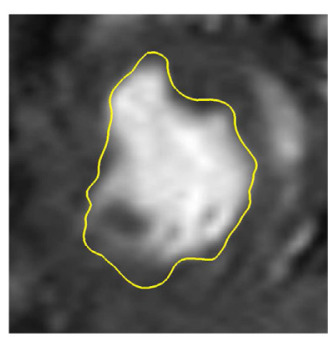

Frame 29

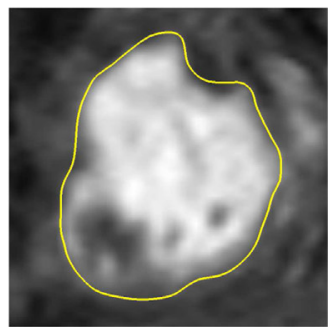

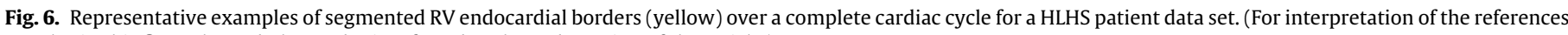
to color in this figure legend, the reader is referred to the web version of the article.)

Table 6

Details of the HLHS patient datasets used in evaluation of the proposed method.

\begin{tabular}{ll}
\hline Description & Value \\
\hline Number of subjects & 23 \\
Patient age & $0.45 \pm 0.30$ years \\
Patient age range & $0.12-1.39$ years \\
Short-axis image size & $(192 \times 114)-(256 \times 200)$ pixels \\
Number of frames $(K)$ & $22-30$ \\
Heart rate & $108-168 \mathrm{bpm}$ \\
Pixel spacing & $(0.27 \times 0.27 \times 5.5)-(0.73 \times 0.73 \times 8) \mathrm{mm}$ \\
\hline
\end{tabular}

\subsubsection{Statistical performance evaluation}

We computed the DM and HD to evaluate the similarities between the manual and automatic segmentations. Table 7 reports the DM and HD values for the HLHS patient data set at ES. We computed the reliability which is given by (number of volumes segmented with DM higher than $d$ )/(total number of volumes), i.e.,
$\mathcal{R} \uparrow(d)=\operatorname{Pr}(D M>d), d \in[0,1]$. Table 7 also reports the reliability at different accuracy levels ( $d=0.70, d=0.75, d=0.80$ ) evaluated over 23 subjects. The analysis shows that $72 \%$ of the automatic contours yielded a Dice score greater than 0.8 . The average DM values for endocardium for each subject in the HLHS patient data sets are depicted in Fig. 7.

We evaluated the performance of the proposed method in estimating the RVESV and RVEF for the HLHS patient data. Fig. 8 reports the Bland-Altman analysis of conformity between manual and automatic contours for RVESV and RVEF estimation. The bias and LOA for RVESV and RVEF were $3.22( \pm 4.38) \mathrm{ml}$ and $-0.12( \pm 0.11)$, respectively.

\section{Discussion}

Our algorithm has the following advantages over prior RV segmentation works: (1) it removes the need for a time-consuming, 
Table 7

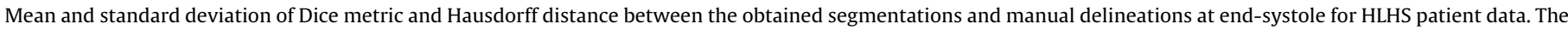
table also reports the reliability $\mathcal{R} \uparrow$ of the proposed method evaluated over 23 HLHS patients for endocardial segmentations.

\begin{tabular}{|c|c|c|c|c|c|}
\hline & Dice metric & Hausdorff distance (mm) & $\mathcal{R} \uparrow(0.70)$ & $\mathcal{R} \uparrow(0.75)$ & $\mathcal{R} \uparrow(0.80)$ \\
\hline Endocardium & $0.82 \pm 0.14$ & $5.60 \pm 2.98$ & 0.83 & 0.79 & 0.72 \\
\hline
\end{tabular}

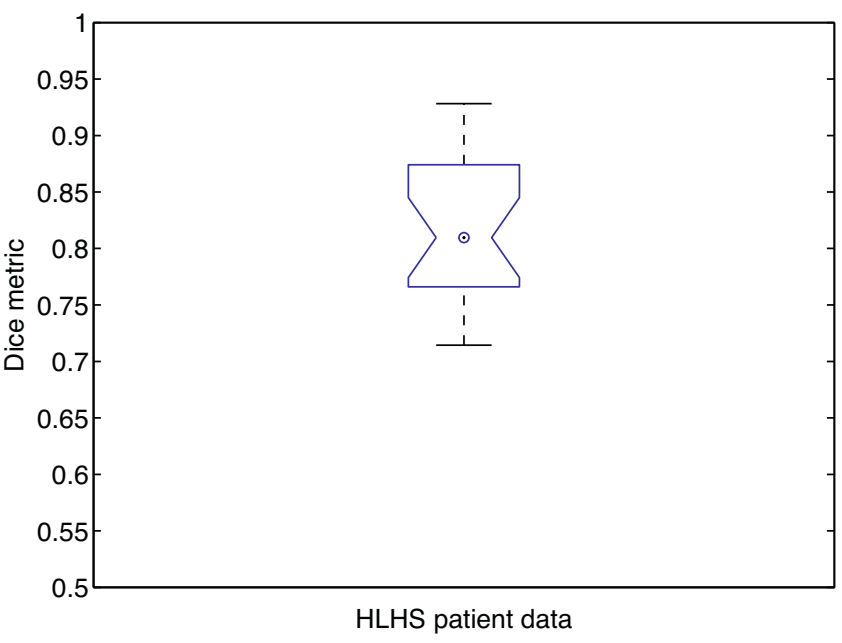

Fig. 7. Boxplot of dice scores for endocardium for the 23 HLHS patient data sets at end-systole. The average number of slices per subject is equal to 7.2.

manually built training set; (2) it does not make prior assumptions as to the distributions of intensity and shape; (3) it provides a sequence of corresponding points over time, a comprehensive input that can be very useful in cardiac applications other than segmentation, e.g., regional wall motion analysis; and (4) it is more flexible for congenital heart disease where the RV undergoes high variations in shape.

An important advantage of our automated method is the ability to measure the volume over the entire cardiac cycle. This allows us to analyse the cardiac function over the entire cardiac in addition to the computation of common clinical measures such as RVEF.

The average size (mean \pm standard deviation) of the moving mesh grid were $(89.1 \times 103.1) \pm(27.7 \times 30.9)$ pixels for the RVSC data. The grid size was smaller for the infant HLHS patient data: $(81.3 \times 83.1) \pm(25.9 \times 26.2)$. The step-then-correct optimization algorithm took an average of $14.7 \pm 9.1$ iterations to converge, and $85 \%$ of the cases converged within a maximum number of iterations equal to 30 .
Table 8

Clinical parameters (mean \pm standard deviation) values using the proposed method, manual contours compared to the reference normal values [43] for adults. The values for our study are different from normal values since the participants are of various heart conditions.

\begin{tabular}{llcc}
\hline Measurement & Proposed method & Manual & Normal values \\
\hline $\operatorname{RVEDV}(\mathrm{ml})$ & N/A & $133 \pm 48$ & $144 \pm 23$ \\
$\operatorname{RVESV}(\mathrm{ml})$ & $73 \pm 39$ & $66 \pm 39$ & $50 \pm 14$ \\
$\operatorname{RVEF}$ & $0.48 \pm 0.12$ & $0.52 \pm 0.13$ & $0.66 \pm 0.06$ \\
$\operatorname{RVM}(\mathrm{g})$ & N/A & $48 \pm 14$ & $48 \pm 13$ \\
\hline
\end{tabular}

Limitations of our study were the lack of a gold standard and the limited number of test subjects. The proposed study assumes manual contours as the gold standard and the automatic contours were compared against manual contours to evaluate the performance of the algorithm. The algorithm was tested only over a data set of 48 adults and a data set of 23 HLHS infants after their initial surgery. Another limitation of our method is that it requires manual contouring of one time frame for a given slice position. Although more time-consuming than automated methods, the method allows for greater accuracy through the remainder of the cardiac cycle.

Although the proposed method does not impose any shape constraints for the heart, its performance depends on the image quality. The presence of any artefacts, including fat, will disrupt the point correspondence mapping. Measuring the global agreement between the manual and automatic contours, the Dice score will not correctly reflect any local imperfections. However, the Hausdorff distance is always higher than or equal to any local point error: it evaluates for each point on the contour the distance between the point and its closest point on the ground truth. The proposed method does not include any temporal characteristics of the cardiac motion in computing the segmentations.

Table 8 reports the mean and standard deviation values for clinical parameter values estimated from the RSVC data and for reference normal value for adults [43]. The RVEF and RVESV values computed using the automatic method is significantly different from the reference normal values. The patients from the RVSC data set have various heart conditions, and therefore, the mean RVEF is

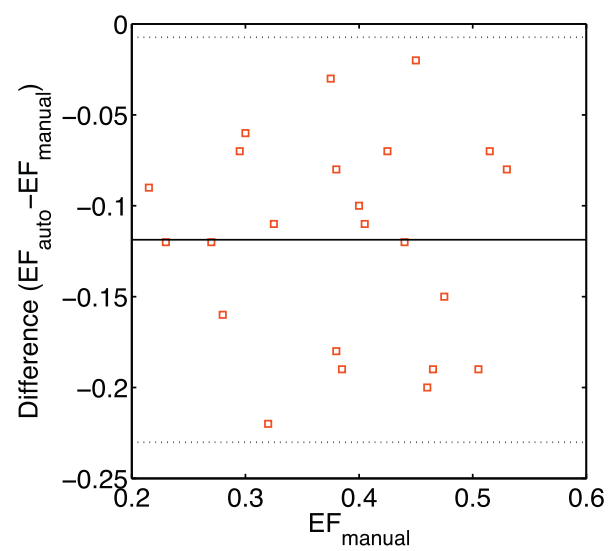

(b) Ejection Fraction

Fig. 8. Comparison of the clinical parameters computed based on the manual and automatic contours using Bland-Altman analysis. 


\section{Table 9}

Normalized clinical parameters (mean \pm standard deviation) values with respect to body surface area (BSA) using the proposed method, manual contours compared to the reference normal values for children [44].

\begin{tabular}{llcl}
\hline Measurement & $\begin{array}{l}\text { Proposed } \\
\text { method }\end{array}$ & Manual & $\begin{array}{l}\text { Normal } \\
\text { values [44] }\end{array}$ \\
\hline RVEDV/BSA $\left(\mathrm{ml} / \mathrm{m}^{2}\right)$ & N/A & $124.5 \pm 29.5$ & $70 \pm 11$ \\
$\operatorname{RVSV} / \mathrm{BSA}\left(\mathrm{ml} / \mathrm{m}^{2}\right)$ & $38 \pm 10$ & $53 \pm 10$ & $43 \pm 7$ \\
$\operatorname{RVEF}$ & $0.35 \pm 0.10$ & $0.44 \pm 0.10$ & $\mathrm{~N} / \mathrm{A}$ \\
\hline
\end{tabular}

lower than the reference normal value. Also, the standard deviation values are higher than the reference normal values.

Table 9 reports the mean and standard deviation values for normalized clinical parameter values estimated from the HLHS data and the corresponding reference normal values for children. The proposed method was evaluated over infants with HLHS whereas the normal values were based on children with normal heart condition.

\section{Conclusion}

In this study, we presented a semi-automated method based on a 2D moving mesh approach to segment the RV from 4D cardiac MR images. The proposed method computes point-to-point correspondences within a cardiac sequence via grid generation. Given a user-provided segmentation of a single frame in the sequence, the proposed method segments both endocardial and epicardial borders of the RV, and does not require a training data set. Evaluated quantitatively over the 48 subjects of the MICCAI 2012 RV segmentation challenge data set, the proposed method yielded average Dice scores of 0.79 and 0.84 , respectively, for endocardial and epicardial segmentations. The proposed method also yielded an average Dice score of 0.81 over 23 hypoplastic left heart syndrome patients, which demonstrates the robustness of the algorithm.

\section{Conflict of interest statement}

MN and KP have submitted a provisional patent application based on the proposed RV segmentation approach. Authors declare that they have no other competing interests.

\section{Acknowledgements}

Authors wish to thank Servier Canada Inc. for the grant which supported this work.

\section{References}

[1] Simon MA. Assessment and treatment of right ventricular failure. Nat Revis Cardiol 2013;10(4):204-18.

[2] Andersen HR, Falk E, Nielsen D. Right ventricular infarction: frequency, size and topography in coronary heart disease: a prospective study comprising 107 consecutive autopsies from a coronary care unit. J Am Coll Cardiol 1987:10(6):1223-32.

[3] Palevsky H, Fishman A. Chronic cor pulmonale: etiology and management. J Am Med Assoc 1990;263(17):2347-53.

[4] Haddad F, Hunt SA, Rosenthal DN, Murphy DJ. Right ventricular function in cardiovascular disease part I: anatomy physiology aging and functional assessment of the right ventricle. Circulation 2008;117(11):1436-48.

[5] Bristow MR, Zisman LS, Lowes BD, Abraham WT, Badesch DB, Groves BM, et al. The pressure-overloaded right ventricle in pulmonary hypertension. CHEST J 1998;114:101S-6S

[6] Humbert M, Sitbon O, Simonneau G. Treatment of pulmonary arterial hypertension. N Engl J Med 2004;351(14):1425-36.

[7] Woodard PK, Bluemke DA, Cascade PN, Finn JP, Stillman AE, Higgins CB, et al. ACR practice guideline for the performance and interpretation of cardiac magnetic resonance imaging (MRI). J Am Coll Radiol 2006;3(9):665-76.

[8] Benza R, Biederman R, Murali S, Gupta H. Role of cardiac magnetic resonance imaging in the management of patients with pulmonary arterial hypertension. J Am Coll Cardiol 2008;52(21):1683-92.
[9] Ghio S, Gavazzi A, Campana C, Inserra C, Klersy C, Sebastiani R, et al. Independent and additive prognostic value of right ventricular systolic function and pulmonary artery pressure in patients with chronic heart failure. J Am Coll Cardiol 2001;37(1):183-8.

[10] Mehta SR, Eikelboom JW, Natarajan MK, Diaz R, Yi C, Gibbons RJ, et al. Impact of right ventricular involvement on mortality and morbidity in patients with inferior myocardial infarction. J Am Coll Cardiol 2001;37(1):37-43.

[11] Bai W, Shi W, Wang H, Peters NS, Rueckert D. Multi-atlas based segmentation with local label fusion for right ventricle MR images. In: Medical Image Computing and Computer-Assisted Intervention, Workshop on RV Segmentation Challenge in Cardiac MRI. 2012.

[12] Grosgeorge D, Petitjean C, Ruan S, Caudron J, Dacher J-N. Right ventricle segmentation by graph cut with shape prior. In: Medical Image Computing and Computer-Assisted Intervention, Workshop on RV Segmentation Challenge in Cardiac MRI. 2012.

[13] Maier OMO, Carretero DJ, Lleo AS, Ledesma-Carbayo MJ. Right-ventricle segmentation with 4D region-merging graph cuts in MR. In: Medical Image Computing and Computer-Assisted Intervention, Workshop on RV Segmentation Challenge in Cardiac MRI. 2012.

[14] Nambakhsh CM, Rajchl M, Yuan J, Peters TM, Ben Ayed I. Rapid automated 3D endocardium right ventricle segmentation in MRI via convex relaxation and distribution matching. In: Medical Image Computing and Computer-Assisted Intervention, Workshop on RV Segmentation Challenge in Cardiac MRI. 2012.

[15] Ou Y, Doshi J, Erus G, Davatzikos C. Multi-atlas segmentation of the cardiac MR right ventricle. In: Medical Image Computing and Computer-Assisted Intervention, Workshop on RV Segmentation Challenge in Cardiac MRI. 2012.

[16] Petitjean C, Zuluaga M, Bai W, Dacher J, Grosgeorge JCD, Ruan S, et al. Right ventricle segmentation from cardiac MRI: a collation study. Med Image Anal $2015 ; 19$

[17] Wang C-W, Peng C-W, Chen H-C. A simple and fully automatic right ventricle segmentation method for 4-dimensional cardiac MR images. In: Medical Image Computing and Computer-Assisted Intervention, Workshop on RV Segmentation Challenge in Cardiac MRI. 2012.

[18] Zuluaga MA, Cardoso MJ, Ourselin S. Automatic right ventricle segmentation using multi-label fusion in cardiac MRI. In: Medical Image Computing and Computer-Assisted Intervention, Workshop on RV Segmentation Challenge in Cardiac MRI. 2012

[19] Nambakhsh CM, Peters TM, Islam A, Ayed IB. Right ventricle segmentation with probability product kernel constraints. In: Medical Image Computing and Computer-Assisted Intervention (MICCAI). 2013.

[20] Caudron J, Fares J, Lefebvre V, Vivier P-H, Petitjean C, Dacher J-N. Cardiac MR assessment of right ventricular function in acquired heart disease: factors of variability. Acad Radiol 2012;19(8):991-1002.

[21] Ben Ayed I, Lu Y, Li S, Ross IG. Left ventricle tracking using overlap priors. In: Medical Image Computing and Computer-Assisted Intervention (MICCAI), Part I. 2008, p. 1025-33.

[22] Ben Ayed I, mei Chen H, Punithakumar K, Ross I, Li S. Max-flow segmentation of the left ventricle by recovering subject-specific distributions via a bound of the Bhattacharyya measure. Med Image Anal 2012;16(1):87-100.

[23] Feng C, Li C, Zhao D, Davatzikos C, Litt H. Segmentation of the left ventricle using a distance regularized two-layer level set approach. In: Medical Image Computing and Computer-Assisted Intervention (MICCAI). 2013.

[24] Nambakhsh CM, Yuan J, Punithakumar K, Goela A, Rajchl M, Peters TM, et al. Left ventricle segmentation in MRI via convex relaxed distribution matching. Med Image Anal 2013;17(8):1010-24.

[25] Paragios N. A level set approach for shape-driven segmentation and tracking of the left ventricle. IEEE Trans Med Imaging 2003;22(6):773-6.

[26] Petitjean C, Dacher J-N. A review of segmentation methods in short axis cardiac MR images. Med Image Anal 2011;15(2):169-84.

[27] Punithakumar K, Ben Ayed I, Islam A, Ross IG, Li S. Tracking endocardial motion via multiple model filtering. IEEE Trans Biomed Eng 2010;57(8):2001-10.

[28] Zhuang X, Rhode K, Arridge S, Razavi R, Hill D, Hawkes D, et al. An atlasbased segmentation propagation framework using locally affine registration - application to automatic whole heart segmentation. In: Metaxas D, editor Medical image computing and computer-assisted intervention, vol. 5242.2008. p. 425-33.

[29] Lotjonen J, Kivisto S, Koikkalainen J, Smutek D, Lauerma K. Statistical shape model of atria ventricles, and epicardium from short- and long-axis MR images. Med Image Anal 2004;8(3):371-86.

[30] Lorenzo-Valdes M, Sanchez-Ortiz GI, Elkington AG, Mohiaddin RH, Rueckert D. Segmentation of 4D cardiac MR images using a probabilistic atlas and the EM algorithm. Med Image Anal 2004;8(3):255-65.

[31] Mitchell S, Lelieveldt B, van der Geest R, Bosch H, Reiber J, Sonka M. Multistage hybrid active appearance model matching: segmentation of left and right ventricles in cardiac MR images. IEEE Trans Med Imaging 2001;0(5):415-23.

[32] Ordas S, Boisrobert L, Huguet M, Frangi AF. Active shape models with invariant optimal features (IOF-ASM): application to cardiac MRI segmentation. Computers in cardiology, vol. 30; 2003. p. 633-6.

[33] Zhang H, Wahle A, Johnson R, Scholz T, Sonka M. 4-D cardiac MR image analysis: left and right ventricular morphology and function. IEEE Trans Med Imaging 2010;29(2):350-64

[34] Ringenberg J, Deo M, Devabhaktuni V, Berenfeld O, Boyers P, Gold J. Fast, accurate, and fully automatic segmentation of the right ventricle in short-axis cardiac MRI. Comput Med Imaging Graph 2014;38(3):190-201.

[35] Li B, Young AA, Cowan BR. Gpu accelerated non-rigid registration for the evaluation of cardiac function. In: Metaxas D, editor. Medical Image Computing 
and Computer-Assisted Intervention (MICCAI), vol. 5242 of Lecture Notes in Computer Science. Berlin, Heidelberg: Springer; 2008. p. 880-7.

[36] Li B, Liu Y, Occleshaw C, Cowan B, Young A. In-line automated tracking for ventricular function with magnetic resonance imaging. J Am Coll Cardiol: Cardiovasc Imaging 2010;3(8):860-6.

[37] Liu J, Ph.D. thesis New development of the deformation method. Department of Mathematics, The University of Texas at Arlington; 2006.

[38] Punithakumar K, Noga M, Boulanger P. Cardiac right ventricular segmentation via point correspondence. In: International Conference of the IEEE Engineering in Medicine and Biology Society. 2013. p. 4010-3.

[39] Chen H-m, Goela A, Garvin GJ, Li S. A parameterization of deformation fields for diffeomorphic image registration and its application to myocardial delineation. In: Jiang T, editor. MICCAI 2010, vol. 6361. 2010. p. 340-8.
[40] Goshtasby A. Image registration: Principles, Tools and Methods, London: Springer; 2012

[41] Zhou X. On uniqueness theorem of a vector function. Prog Electromagn Res 2006;65:93-102.

[42] Huttenlocher D, Klanderman G, Rucklidge W. Comparing images using the Hausdorff distance. IEEE Trans Pattern Anal Mach Intell 1993;5(9):850-63.

[43] Maceira AM, Prasad SK, Khan M, Pennell DJ. Reference right ventricular systolic and diastolic function normalized to age gender and body surface area from steady-state free precession cardiovascular magnetic resonance. Eur Heart J 2006;27(23):2879-88.

[44] Lorenz C. The range of normal values of cardiovascular structures in infants children and adolescents measured by magnetic resonance imaging. Pediatr Cardiol 2000;21(1):37-46 